\title{
A new proof of Kelley's Theorem
}

\author{
by
}

\author{
Siu-Ah Ng (Hull)
}

\begin{abstract}
Kelley's Theorem is a purely combinatorial characterization of measure algebras. We first apply linear programming to exhibit the duality between measures and this characterization for finite algebras. Then we give a new proof of the Theorem using methods from nonstandard analysis.
\end{abstract}

First some notation and definitions. $\mathcal{B}$ always denotes a Boolean algebra of the form $(\mathcal{B}, 0,1,+, \cdot,-)$ with the induced ordering $\leq$. We sometimes write $\sum$ and $\prod$ for + and $\cdot$, especially when the operations are infinitary. By a measure $\mu$ on a subalgebra $\mathcal{A} \subseteq \mathcal{B}$ we mean a finitely additive monotone function $\mu: \mathcal{A} \rightarrow[0,1]$ so that $\mu(0)=0$ and $\mu(1)=1$. A Boolean algebra $\mathcal{B}$ is called a measure algebra if there is a measure $\mu: \mathcal{B} \rightarrow[0,1]$ which is strictly positive, i.e. $\mu(b)=0$ iff $b=0$. A $\sigma$-algebra $\mathcal{B}$ is called a $\sigma$-measure algebra if there is such a $\sigma$-additive $\mu$. Following $[\mathrm{K}]$, we define for $\mathcal{A} \subseteq \mathcal{B}$ the intersection number of $\mathcal{A}$ as

$$
\alpha(\mathcal{A})=\inf \left\{\widehat{\alpha}\left(a_{1}, \ldots, a_{n}\right): a_{1}, \ldots, a_{n} \in \mathcal{A}, n<\omega\right\},
$$

where $\widehat{\alpha}\left(a_{1}, \ldots, a_{n}\right)=n^{-1} \max \left\{|I|: I \subseteq\{1, \ldots, n\}, \prod_{i \in I} a_{i} \neq 0\right\}$. (The $a_{i}$ 's are not necessarily distinct.) We also define the measure number of $\mathcal{A}$ as

$$
\begin{aligned}
\beta(\mathcal{A})=\sup \{r \in[0,1]: \text { there is a measure } \mu \text { on the subalgebra } \\
\text { generated by } \mathcal{A} \text { such that } \mu(a) \geq r \text { for all } a \in \mathcal{A}\} .
\end{aligned}
$$

A $\sigma$-algebra $\mathcal{B}$ is said to be weakly $\omega$-distributive if given $\left\{b_{i j}: i, j<\omega\right\}$ such that $b_{i, j} \geq b_{i j+1}$, then

$$
\sum_{i=0}^{\omega} \prod_{j=0}^{\omega} b_{i j}=\prod_{n=0}^{\omega} \sum_{i=0}^{\omega} b_{i f_{n}(i)}
$$

for some $f_{n}: \omega \rightarrow \omega$ such that $f_{n} \leq f_{n+1}$ (i.e. $\forall i, f_{n}(i) \leq f_{n+1}(i)$ ). 
We assume some basic knowledge of nonstandard analysis (cf. e.g. [HL] and $[\mathrm{L}])$ and work in a $\aleph_{1}$-saturated nonstandard universe that has the enlargement property (i.e. every standard set has a hyperfinite extension). We use nonstandard analysis and the duality theorem for linear programming to prove the following theorem of J. L. Kelley. See $[K]$ or $[F]$ for a standard proof.

Kelley's TheOrem. (1) A Boolean algebra $\mathcal{B}$ is a measure algebra iff (*) there are $\mathcal{A}_{n} \subset \mathcal{B}$ such that $\alpha\left(\mathcal{A}_{n}\right)>0$ and $\mathcal{B}=\bigcup_{n<\omega} \mathcal{A}_{n} \cup\{0\}$.

(2) $A \sigma$-algebra $\mathcal{B}$ is a $\sigma$-measure algebra iff $\mathcal{B}$ is weakly $\omega$-distributive and also satisfies $(*)$.

The proof is based on the following lemma, which exhibits the duality between measures and Kelley's characterization.

Lemma. Let $\mathcal{A} \subseteq \mathcal{B}$ such that $\mathcal{A}$ is finite. Then $\alpha(\mathcal{A})=\beta(\mathcal{A})$ and both the infimum and supremum in the definitions of $\alpha$ and $\beta$ are attained.

Proof. Write $\mathcal{A}=\left\{a_{0}, \ldots, a_{n}\right\}$, and identify the finite Boolean algebra generated by $\mathcal{A}$ as a power set algebra $\mathcal{P}(X)$ for some $X=\left\{p_{0}, \ldots, p_{r}\right\}$.

For $i \leq r, j \leq n$, define

$$
m_{i j}= \begin{cases}1 & \text { if } p_{i} \in a_{j} \\ 0 & \text { otherwise }\end{cases}
$$

and write $M=\left[m_{i j}\right]$, an $(r+1) \times(n+1)$ binary matrix.

Claim 1. (a) $\alpha(\mathcal{A})$ is the minimal $\alpha \in \mathbb{R}$ such that

$$
M \cdot\left[\begin{array}{c}
x_{0} \\
\vdots \\
x_{n}
\end{array}\right] \leq\left[\begin{array}{c}
\alpha \\
\vdots \\
\alpha
\end{array}\right]
$$

for some $x_{0}+\ldots+x_{n} \geq 1, x_{0}, \ldots, x_{n} \geq 0$.

(b) $\alpha(\mathcal{A})=\widehat{\alpha}\left(a_{i_{0}}, \ldots, a_{i_{l}}\right)$ for some $a_{i_{0}}, \ldots, a_{i_{l}} \in \mathcal{A}$.

Proof. Let $\left(\alpha_{0}, \ldots, \alpha_{n}, \alpha\right) \in[0, \infty)^{n+2}$ be an extreme point in a region determined by some of the $r+2$ hyperplanes

$$
\begin{gathered}
m_{i 0} x_{0}+\ldots+m_{i n} x_{n}=x_{n+1}, \quad i=0, \ldots, r, \\
x_{0}+\ldots+x_{n}=1,
\end{gathered}
$$

with the property that $\alpha$ is minimal.

Then this is the minimal $\alpha$ such that $M \cdot\left[x_{0}, \ldots, x_{n}\right]^{T} \leq \alpha$ among all $x_{0}, \ldots, x_{n} \geq 0$ satisfying $x_{0}+\ldots+x_{n} \geq 1$. Since the coefficient of each one of the $x_{i}$ is either 0 or 1 , the extreme point is a rational point of the form

$$
\left(\alpha_{0}, \ldots, \alpha_{n}, \alpha\right)=\left(s_{0} / s, \ldots, s_{n} / s, \alpha\right),
$$

where $s_{0}, \ldots, s_{n} \in \mathbb{N}$ and $s_{0}+\ldots+s_{n}=s$. 
Any such rational point $\left(s_{0} / s, \ldots, s_{n} / s\right)$ is in one-to-one correspondence with the following sequence from $\mathcal{A}$ :

$$
\sigma=\left(a_{0} \ldots a_{0} a_{1} \ldots a_{1} \ldots a_{n} \ldots a_{n}\right),
$$

where $a_{0}$ repeats $s_{0}$ times, $a_{1}$ repeats $s_{1}$ times, etc.

Moreover, for the above extreme point, we have $\widehat{\alpha}(\sigma)=\alpha$, and for any other sequence $\tau$ from $\mathcal{A}$, we have $\widehat{\alpha}(\tau) \geq \alpha$. Therefore $\alpha(\mathcal{A})=\alpha$ and (a) is proved. Since $\alpha(\mathcal{A})=\widehat{\alpha}(\sigma)$, (b) holds as well.

Claim 2. (a) $\beta(\mathcal{A})$ is the maximal $\beta \in \mathbb{R}$ such that

$$
M^{T} \cdot\left[\begin{array}{c}
y_{0} \\
\vdots \\
y_{r}
\end{array}\right] \geq\left[\begin{array}{c}
\beta \\
\vdots \\
\beta
\end{array}\right]
$$

for some $y_{0}+\ldots+y_{r} \leq 1, y_{0}, \ldots, y_{r} \geq 0$.

(b) $\beta(\mathcal{A})$ is attained by some measure on the subalgebra generated by $\mathcal{A}$.

Proof. Similar to Claim 1, the maximal $\beta$ satisfying the matrix inequality is given by some rational point $\left(\beta_{0}, \ldots, \beta_{r}, \beta\right)$. This corresponds to the measure that assigns weights $\beta_{0}, \ldots, \beta_{r}$ to $p_{0}, \ldots, p_{r}$ respectively. Conversely, any such weights $\beta_{0}, \ldots, \beta_{r}$ satisfy the matrix inequality for some $\beta$, thus the claim is proved.

The linear programming problems in the above two claims are dual to each other, so $\alpha(\mathcal{A})=\beta(\mathcal{A})$ and the lemma is proved.

Corollary. $\alpha(\mathcal{A}) \geq \beta(\mathcal{A})$ for arbitrary $\mathcal{A} \subseteq \mathcal{B}$.

Proof. $\alpha(\mathcal{A})=\inf \alpha\left(\mathcal{A}_{0}\right)=\inf \beta\left(\mathcal{A}_{0}\right) \geq \beta(\mathcal{A})$, where the infimum is taken over all finite $\mathcal{A}_{0} \subseteq \mathcal{A}$.

Proof of Kelley's Theorem $(1) .(\Rightarrow)$. Let $\mu: \mathcal{B} \rightarrow[0,1]$ be a strictly positive measure and let $\mathcal{A}_{n}=\left\{b \in \mathcal{B}: \mu(b) \geq 2^{-n}\right\}$. Then $\mathcal{B}=\bigcup_{n<\omega} \mathcal{A}_{n} \cup\{0\}$. Since $\beta\left(\mathcal{A}_{n}\right) \geq 2^{-n}$, so by the Corollary, $\alpha\left(\mathcal{A}_{n}\right)>0$.

$(\Leftarrow)$. Suppose $\mathcal{B}=\bigcup_{n<\omega} \mathcal{A}_{n} \cup\{0\}$ and $\alpha\left(\mathcal{A}_{n}\right)>0$. Use the enlargement property and let $\mathcal{B}^{\prime}$ be a hyperfinite algebra such that $\mathcal{B} \subseteq \mathcal{B}^{\prime} \subseteq{ }^{*} \mathcal{B}$. Let $\mathcal{B}_{n}=\mathcal{B}^{\prime} \cap{ }^{*} \mathcal{A}_{n}$. It follows from $\left\{{ }^{*} a: a \in \mathcal{A}_{n}\right\} \subseteq \mathcal{B}_{n} \subseteq{ }^{*} \mathcal{A}_{n}$ and the transfer principle that ${ }^{*} \alpha\left(\mathcal{B}_{n}\right) \approx \alpha\left(\mathcal{A}_{n}\right)>0$ and both are noninfinitesimal. By transferring the Lemma, ${ }^{*} \alpha\left(\mathcal{B}_{n}\right)={ }^{*} \beta\left(\mathcal{B}_{n}\right)$, and hence there is an internal measure $\nu_{n}$ on the subalgebra generated by $\mathcal{B}_{n}$ such that for each $b \in \mathcal{B}_{n}$, $\nu_{n}(b) \geq{ }^{*} \alpha\left(\mathcal{B}_{n}\right)>0$, noninfinitesimal. For each $m<\omega$, there is an internal measure $\nu$ on $\mathcal{B}^{\prime}$ such that $\nu \geq 2^{-n} \nu_{n}$ on $\mathcal{B}_{n}$ for all $n<m$. So by $\aleph_{1^{-}}$ saturation, there is an internal measure $\nu$ on $\mathcal{B}^{\prime}$ such that for all $n<\omega$, $\nu \geq 2^{-n} \nu_{n}$ on $\mathcal{B}_{n}$. Let $\mu={ }^{\circ} \nu \uparrow \mathcal{B}$, i.e. $\forall b \in \mathcal{B}, \mu(b)={ }^{\circ} \nu\left({ }^{*} b\right)$. Then $\mu$ is a strictly positive measure on $\mathcal{B}$. 
Proof of Kelley's Theorem $(2) .(\Rightarrow)$. Let $\mu: \mathcal{B} \rightarrow[0,1]$ be a strictly positive $\sigma$-measure. By $(1),(*)$ holds. To show the weak $\omega$ distributivity, let $\left\{b_{i j}\right\}$ be such that $b_{i j} \geq b_{i, j+1}$. For each $n$ and $i<\omega$, let $f_{n}(i)=$ least $j$ such that

$$
\mu\left(b_{i j}-\prod_{k=0}^{\omega} b_{i k}\right) \leq 1 / 2^{n+i+1} .
$$

Then $\mu\left(\sum_{i=0}^{\omega} b_{i f_{n}(i)}-\sum_{i=0}^{\omega} \prod_{j=0}^{\omega} b_{i j}\right) \leq 1 / 2^{n}$. Hence

$$
\prod_{n=0}^{\omega} \sum_{i=0}^{\omega} b_{i f_{n}(i)}=\sum_{i=0}^{\omega} \prod_{j=0}^{\omega} b_{i j} .
$$

$(\Leftarrow)$. Let $\mathcal{B}^{\prime}$ be a hyperfinite algebra so that $\mathcal{B} \subseteq \mathcal{B}^{\prime} \subseteq{ }^{*} \mathcal{B}$. Let $\nu$ be the internal measure given by the proof in $(1)$. Then ${ }^{\circ} \nu(\bar{b})>0$ for each $b \in \mathcal{B}^{\prime} \cap{ }^{*} \mathcal{A}_{n}$. Let

$\mathcal{D}=\left\{b \in \mathcal{B}^{\prime}:\right.$ there is a decreasing sequence $\left\{c_{n}\right\}_{n<\omega}$ from $\mathcal{B}$

$$
\text { so that } \left.\prod_{n=0}^{\omega} c_{n}=0 \text { and each }{ }^{*} c_{n} \geq b\right\} \text {. }
$$

Note that $\prod_{n=0}^{\omega} c_{n}$ is defined for the $\sigma$-algebra $\mathcal{B}$, while in general, $\mathcal{B}^{\prime}$ is not a $\sigma$-algebra, and $\left\{{ }^{*} c_{n}\right\}_{n<\omega}$ may have nonzero lower bounds in $\mathcal{B}^{\prime}$.

Define $r=\sup \left\{{ }^{\circ} \nu(b): b \in \mathcal{D}\right\}$.

Claim. $r$ is attained by some $a^{\prime} \in \mathcal{D}$. In other words, there are $a^{\prime} \in \mathcal{B}^{\prime}$ and decreasing $d_{n} \in \mathcal{B}$ so that $\prod_{n=0}^{\omega} d_{n}=0$, each ${ }^{*} d_{n} \geq a^{\prime}$ and $r \approx \nu\left(a^{\prime}\right)$.

Proof. Choose $b_{0}, b_{1}, \ldots$ from $\mathcal{D}$ so that ${ }^{\circ} \nu\left(b_{i}\right) \rightarrow r$. For each $i$, choose $c_{i n} \in \mathcal{B}(n<\omega)$ so that $c_{i n}$ decreases to 0 as $n \rightarrow \omega$ and each ${ }^{*} c_{i n} \geq b_{i}$. By the weak $\omega$-distributivity, $\sum_{i=0}^{\omega} \prod_{j=0}^{\omega} c_{i j}=\prod_{n=0}^{\omega} \sum_{i=0}^{\omega} c_{i f_{n}(i)}$ for some $f_{n}: \omega \rightarrow \omega$ such that $f_{n} \leq f_{n+1}$. The left hand side equals 0 . Write $d_{n}=\sum_{i=0}^{\omega} c_{i f_{n}(i)}$. Then $\prod_{n=0}^{\omega} d_{n}=0$. Note also that $d_{n} \in \mathcal{B}$ and decreases. For any $n, m$, there is $a \in \mathcal{B}^{\prime}$ such that $b_{0}+\ldots+b_{m}=a \leq{ }^{*} d_{n}$, so by $\aleph_{1^{-}}$ saturation, there is an internal $a^{\prime} \in \mathcal{B}^{\prime}$ such that $b_{i} \leq a^{\prime} \leq{ }^{*} d_{n}$ for all $i, n<\omega$ (so in particular $a^{\prime} \in \mathcal{D}$ ), and ${ }^{\circ} \nu\left(a^{\prime}\right) \geq \sup _{i<\omega}{ }^{\circ} \nu\left(b_{i}\right)$. Hence ${ }^{\circ} \nu\left(a^{\prime}\right)=r$. The claim is proved.

Notice that $\nu\left(a^{\prime}\right) \not 1$. Suppose otherwise; then for each $n<\omega,{ }^{\circ} \nu\left({ }^{*} d_{n}\right)$ $=1$, so ${ }^{\circ} \nu\left(1-{ }^{*} d_{n}\right)=0$, and by the strict positivity of ${ }^{\circ} \nu \uparrow \mathcal{B}$, it follows that $d_{n}=1$, contradicting $\prod_{n=0}^{\omega} d_{n}=0$.

Now define the internal measure $\mu(b)=\nu\left(b-a^{\prime}\right) /\left(1-\nu\left(a^{\prime}\right)\right), b \in \mathcal{B}^{\prime}$. For $b \in \mathcal{B}$, write ${ }^{\circ} \mu(b)={ }^{\circ}\left(\mu\left({ }^{*} b\right)\right)$. Then ${ }^{\circ} \mu$ is a strictly positive $\sigma$-measure on $\mathcal{B}$. To show this, it suffices to verify the following.

(i) If $0 \neq b \in \mathcal{B}$ then ${ }^{\circ} \mu(b)>0$. 
Proof. Let $d_{n}$ decrease to 0 in $\mathcal{B}$ and ${ }^{*} d_{n} \geq a^{\prime}$ for any $n<\omega$, as in the claim. Since $b \neq 0, b$ is not a lower bound of $\left\{d_{n}\right\}$, therefore $b-d_{n} \neq 0$ for some $n$. Then ${ }^{\circ} \nu\left({ }^{*} b-{ }^{*} d_{n}\right)>0$, so ${ }^{\circ} \nu\left({ }^{*} b-a^{\prime}\right)>0$, so ${ }^{\circ} \mu(b)>0$.

(ii) ${ }^{\circ} \mu$ is $\sigma$-additive on $\mathcal{B}$.

Proof. Let $\left\{c_{n}\right\}_{n<\omega}$ be a sequence decreasing to 0 in $\mathcal{B}$. By enlargement, this extends to a sequence $\left\{c_{n}\right\}_{n<\mathrm{H}}$ in $\mathcal{B}^{\prime}$, where $\mathrm{H}$ is an infinite hyperfinite integer, and for convenience, we omit the "**" from ${ }^{*} c_{n}$ for finite $n$. From the definition of $\mathcal{D}, c_{\mathrm{N}} \in \mathcal{D}$ for each infinite $\mathrm{N}$. By $a^{\prime}$ being maximal, for any infinite $\mathrm{N}, \nu\left(c_{\mathrm{N}}+a^{\prime}\right) \approx \nu\left(a^{\prime}\right)$, so $\nu\left(c_{\mathrm{N}}-a^{\prime}\right) \approx 0$. Thus $\mu\left(c_{\mathrm{N}}\right) \approx 0$ for any infinite N. Therefore ${ }^{\circ} \mu\left(c_{n}\right) \rightarrow 0$.

Acknowledgements. I was supported by an SERC Grant during the writing of this paper. I am very grateful to the unknown referee for his careful reading and very helpful suggestions.

\section{References}

[F] D. H. Fremlin, Measure algebras, in: Handbook of Boolean Algebra, Vol. III, J. D. Monk and R. Bonnet (eds.), North-Holland, Amsterdam 1989, 877-980.

[HL] A. Hurd and P. A. Loeb, An Introduction to Nonstandard Real Analysis, Academic Press, New York 1985.

[K] J. L. Kelley, Measures on Boolean algebras, Pacific J. Math. 9 (1959), 1165-1177.

[L] T. L. Lindstrøm, An invitation to nonstandard analysis, in: Nonstandard Analysis and its Applications, N.J. Cutland (ed.), Cambridge University Press, 1988, $1-105$.

DEPARTMENT OF PURE MATHEMATICS

UNIVERSITY OF HULL

HULL, HU6 7RX ENGLAND 